\title{
Relaciones del Perfil Lipídico con Variables Dietéticas, Antropométricas, Bioquímicas, y Otros Factores de Riesgo Cardiovascular en Estudiantes Universitarios
}

\author{
Guido Ulate-Montero, ${ }^{1}$ Aileen Fernández-Ramírez ${ }^{1}$
}

\begin{abstract}
Justificación y objetivos: La enfermedad de las arterias coronarias, al igual que otras enfermedades crónicas, tiene su origen en la infancia y la adolescencia. El objetivo de esta investigación fue determinar las variables antropométricas y bioquímicas, el nivel de actividad física y los componentes de la dieta que se relacionan y además podrían predecir los niveles plasmáticos de colesterol, LDL, HDL y triglicéridos en estudiantes jóvenes costarricenses.
\end{abstract}

\begin{abstract}
Métodos: La muestra estudiada la formaron 110 estudiantes (59 mujeres y 51 hombres) de la Universidad de Costa Rica con edades entre los 17 y 20 años, seleccionados aleatoriamente del total de estudiantes que ingresaron en 1996. Se evaluaron parámetros antropométricos, de la dieta, la bioquímica sanguínea y el consumo de oxígeno. Las relaciones entre el perfil lipídico (variables dependientes) y el resto de variables evaluadas (independientes) se analizaron por medio de coeficientes de correlación de Pearson y modelos de regresión múltiple (stepwise).
\end{abstract}

Resultados: Los niveles de colesterol total y de LDL se relacionan de manera directa y significativa $(\mathrm{p}<0.01)$ con el porcentaje de grasa corporal y los triglicéridos. La relación entre las LDL y el consumo máximo de oxígeno fue inversa $(\mathrm{p}<0.05)$. Los niveles altos de triglicéridos, de ácido úrico, de cintura, de índice de masa corporal $\mathrm{y}$ de ingesta de $\mathrm{B}_{6}$, se relacionaron significativamente $(\mathrm{p}<0.05)$ con concentraciones bajas de HDL. Aproximadamente, un $50 \%\left(\mathrm{R}^{2}=0.459\right)$ de la variabilidad del colesterol, es explicado por el sexo, los niveles plasmáticos de triglicéridos, de HDL y de potasio.

Conclusiones: Se encontró que en individuos jóvenes, el sexo y ciertas variables antropométricas, como el índice de masa corporal, la relación cintura/cadera y el porcentaje de grasa corporal, presentaron las asociaciones más importantes con los niveles séricos de los lípidos y las lipoproteínas evaluados. Además, el consumo máximo de oxígeno, los niveles plasmáticos de ácido úrico y potasio, el consumo de fibra, $\mathrm{B}_{6}$, vitamina $\mathrm{C}$ y ácido fólico también se relacionaron significativamente con el perfil lipídico.

Descriptores: Enfermedad coronaria, factores de riesgo, perfil lipídico, dieta, adolescencia.

Recibido: 26 de febrero de 2001.

Aceptado: 08 de mayo de 2001.

\begin{abstract}
Abreviaturas: DE, desviación estándar; EAC, enfermedad de las arterias coronarias; HDL, lipoproteína de alta densidad; HTA, hipertensión arterial; ICC, índice cintura/cadera; IMC, índice de masa corporal; LDL, lipoproteína de baja densidad; VO2 max, consumo máximo de oxígeno.

Departamento de Fisiología, Facultad de Medicina, Universidad de Costa Rica.
\end{abstract}

Correspondencia: Guido Ulate Montero. Apartado Postal 1300-2050 San Pedro, Costa Rica. Correo electrónico: gulate@ cariari.ucr.ac.cr
Durante las últimas dos décadas, gran cantidad de estudios epidemiológicos y de intervención han establecido el papel de varios factores de riesgo en el desarrollo de la enfermedad de las arterias coronarias (EAC). Estos incluyen: alteraciones del perfil lipídico como niveles elevados de lipoproteínas de baja densidad (LDL) y colesterol o niveles bajos de lipoproteínas de alta densidad (HDL), hipertensión arterial (HTA), consumo de tabaco, obesidad, diabetes mellitus e inactividad física. ${ }^{1-5}$ 
La EAC, al igual que otras enfermedades crónicas, tiene su origen en la infancia y la adolescencia. En un estudio sobre determinantes patobiológicos de la aterosclerosis en jóvenes (PDAY), el examen patológico de la arteria coronaria derecha y de la aorta abdominal, de hombres y mujeres con edades entre los 15 y 34 años, demostró que la aterosclerosis se inicia tempranamente. ${ }^{6}$ Además, se encontró una relación significativa entre la hiperlipidemia y la extensión de la aterosclerosis.

Asimismo, se ha reportado, en jóvenes, un incremento en la severidad de la aterosclerosis coronaria y aórtica conforme aumenta el número de factores de riesgo cardiovascular. ${ }^{7,8}$ Algunos de estos factores existen desde la infancia y tienden a mantenerse durante el crecimiento y en la vida adulta. Se ha demostrado la importancia de dar seguimiento a aquellos niños y jóvenes con niveles plasmáticos altos de colesterol y LDL ya que un gran porcentaje de ellos persistirán presentando niveles alterados en su vida adulta. ${ }^{9-11}$

Después de 50 años de investigación, en adultos, se ha podido concluir que la dieta es una de las causas ambientales más importantes en la producción de la aterosclerosis y sus secuelas, ${ }^{12}$ probablemente debido a su efecto sobre las concentraciones plasmáticas de lípidos. El factor dietético que más afecta la concentración plasmática de colesterol y de LDL es el consumo de grasas saturadas, particularmente las que poseen una cadena de 12 (ácido láurico), 14 (ácido mirístico) y 16 (ácido palmítico) carbonos. ${ }^{13,14}$ También influye la cantidad ingerida de grasas poliinsaturadas y monoinsaturadas; al respecto, se ha reportado que las primeras reducen tanto las LDL como las HDL pero que las segundas parecen reducir solamente las LDL. ${ }^{13}$ La cantidad ingerida de colesterol se ha relacionado positivamente con los niveles plasmáticos de colesterol, pero en mucho menor grado que con la cantidad ingerida de grasas saturadas. ${ }^{15}$

La influencia de la actividad física sobre el perfil lipídico ha sido estudiada ampliamente. Básicamente, se ha descrito un efecto beneficioso del ejercicio regular pues este incrementa los niveles plasmáticos de HDL y disminuye los niveles de triglicéridos. ${ }^{16}$

Sin embargo, también se ha determinado que cuando la EAC ocurre en personas menores de 60 años, existe una fuerte agregación familiar ${ }^{17,18}$ que podría explicar la razón por la que los factores de riesgo suelen presentarse de manera combinada y desde edades tempranas. Por ejemplo, la dislipidemia, la obesidad, la hipertensión arterial y la diabetes mellitus, suelen presentarse juntos en un mismo individuo, muchas veces desde su juventud, como parte del llamado síndrome de resistencia a la insulina o síndrome $\mathrm{X} \cdot{ }^{19,20}$

Interesa conocer los factores que, desde edades tempranas, se relacionan con un perfil lipídico alterado por varias razones: 1) La identificación de dichos factores permitirá un mejor abordaje del problema, especialmente en lo que corresponde al tratamiento y la prevención. 2) El conjunto de factores asociados podría describir uno o varios síndromes con un fondo genético que ayudaría a conocer mejor el origen de la EAC. 3) Algunos de los factores asociados podrían no estar genéticamente determinados, pero debido a la temprana coexistencia con la hiperlipidemia, podrían influir de una manera importante en la evolución de la placa aterosclerótica. Al respecto, se ha descrito que factores como la HTA, la glicosilación proteica, irritantes químicos como el humo del cigarrillo, aminas circulantes vasoactivas, complejos inmunes e infecciones, pueden potenciar una lesión crónica endotelial mínima. ${ }^{12}$

El objetivo de esta investigación fue determinar las variables antropométricas y bioquímicas, el nivel de actividad física y los componentes de la dieta que se relacionan y además podrían predecir los niveles plasmáticos de colesterol, LDL, HDL y triglicéridos en estudiantes jóvenes costarricenses.

\section{Materiales y Métodos}

La muestra incluyó 110 estudiantes (59 mujeres y 51 hombres) de la Universidad de Costa Rica con edades comprendidas entre los 17 y 20 años. La selección se realizó por medio de un muestreo aleatorio simple con base en la lista de estudiantes que ingresaron por primera vez a la Universidad durante el primer semestre de 1996. Se obtuvo consentimiento informado de cada estudiante por escrito. El protocolo de investigación cumplió con los requerimientos de la Comisión de Investigación de la Escuela de Medicina de la Universidad de Costa Rica.

Los sujetos fueron sometidos a un examen físico en el cual se determinó el peso corporal utilizando una balanza médica marca Toledo y la talla por medio de un tallímetro diseñado especialmente para tal efecto. Además se les midió la cintura (tamaño de la parte más angosta del tronco), la cadera (parte más prominente a nivel de los glúteos) y se calculó la relación cintura/cadera (ICC). La adiposidad (\% de grasa) se determinó con un calibrador de grasa (Lange) midiendo los pliegues de tejido subcutáneo tricipital, subescapular, suprailíaco, abdominal, pectoral y del cuadríceps.

También se recolectó una muestra de sangre después de 12 horas de ayuno y en ella se midieron: ácido úrico, glucosa, sodio, potasio y el perfil lipídico: colesterol total, LDL, HDL y triglicéridos. La técnica y los instrumentos utilizados están descritos en una publicación previa. ${ }^{21}$ El consumo máximo de oxígeno ( $\left.\mathrm{Vo}_{2} \max \right)$ se determinó por medio de la prueba de Astrand y Ryhming. ${ }^{22}$ En un cuestionario escrito, previamente validado, se solicitó la información sobre el consumo de tabaco.

La determinación de la ingesta diaria de los diferentes nutrientes se realizó por el método de consumo usual de alimentos, el cual está diseñado para aquellos casos donde el consumo diario es atípico. ${ }^{23,24}$ Básicamente, se preguntó por los alimentos usualmente ingeridos en los diferentes tiempos de comida de un día normal. Se determinó el patrón para un día entre semana. Como complemento a este método se utilizaron foto- 
grafías de porciones comúnmente consumidas, para cuantificar las cantidades de alimentos. ${ }^{25}$

Para el análisis nutricional, los datos se procesaron de forma individual en el programa de cómputo Nutritionist IV. La ingesta de carbohidratos, proteínas, lípidos totales, ácidos grasos saturados, monoinsaturados y poliinsaturados se expresó como el porcentaje de calorías correspondiente a cada uno de estos nutrientes, con respecto al total de calorías ingerido.

Para describir los resultados, se utilizaron la media y desviación estándar (DE) como medidas de tendencia central. Las comparaciones entre sexos se realizaron por medio de la t de student. Para evaluar la asociación entre las diferentes variables antropométricas, bioquímicas, dietéticas y el perfil lipídico, se calcularon los coeficientes de correlación de Pearson y se determinó su significancia estadística. Por último, con el propósito de identificar las asociaciones independientes entre las diversas variables estudiadas y cada una de las cuatro variables del perfil lipídico, se realizó un análisis de regresión múltiple escalonada (stepwise). Debido a que la distribución para los niveles de HDL y triglicéridos no fue normal, se les aplicó una transformación logarítmica. Se trabajó con un nivel de significancia de $\mathrm{p}<0.05$.

\section{Resultados}

En el Cuadro 1 se presenta la estadística descriptiva para los parámetros antropométricos, la bioquímica sanguínea y el $\mathrm{Vo}_{2}$ max para los hombres, las mujeres y el total de la muestra. El peso, la talla, la cintura, el ICC, el $\mathrm{VO}_{2} \max$, los niveles plas- máticos de ácido úrico y potasio fueron significativamente mayores en los hombres que en las mujeres. El porcentaje de grasa corporal, los niveles plasmáticos de colesterol total y LDL fueron significativamente mayores en las mujeres.

En el Cuadro 2 se muestran las medias y DE del consumo diario de diferentes nutrientes. La cantidad ingerida de calorías, de $\mathrm{B}_{12}$, de $\mathrm{B}_{6} \mathrm{y}$ ácido fólico fueron significativamente mayores en los hombres que en las mujeres. Cabe destacar los bajos consumos de fibra tanto en hombres como en mujeres, las altas ingestas absolutas de colesterol en hombres (>300 mg) y un porcentaje de calorías proveniente de las grasas totales superior al límite recomendado (30\%) en las mujeres. Los porcentajes de calorías provenientes de carbohidratos, proteínas, grasas saturadas, monoinsaturadas y poliinsaturadas están dentro de los límites recomendados. ${ }^{26}$

El porcentaje de hombres y mujeres fumadores fue $15.7 \%$ y $3.4 \%$ respectivamente, y en el total de la muestra fue $9.1 \%$.

Los coeficientes de correlación entre los niveles plasmáticos de colesterol total, LDL, HDL, triglicéridos y las diferentes variables antropométricas, bioquímicas, dietéticas y el $\mathrm{VO}_{2}$ max se muestran en el Cuadro 3. Los niveles de colesterol total se relacionaron de manera directa y significativa con el porcentaje de grasa, los niveles de LDL y de triglicéridos y de manera inversa con el ICC. Los niveles de LDL se relacionaron de igual forma pero además mostraron una asociación inversa significativa con el $\mathrm{Vo}_{2} \max$. Niveles altos de triglicéridos, ácido úrico, ICC, cintura, IMC y de ingesta de $\mathrm{B}_{6}$ se relacionaron, en forma significativa, con concentraciones

\begin{tabular}{|c|c|c|c|c|c|c|}
\hline \multirow{3}{*}{ Característica } & \multicolumn{5}{|c|}{$\begin{array}{c}\text { Cuadro } 1 \\
\text { aracterísticas de los estudiantes universitarios según sexo. } \\
\text { Universidad de Costa Rica, } 1996\end{array}$} & \multirow{3}{*}{$\begin{array}{l}\text { 110) } \\
\text { Rango }\end{array}$} \\
\hline & \multicolumn{2}{|c|}{ Hombres $(n=51)$} & \multicolumn{2}{|c|}{ Mujeres $(n=59)$} & Total $(n=110)$ & \\
\hline & Promedio $\pm \mathrm{DE}$ & Rango & Promedio $\pm \mathrm{DE}$ & Rango & Promedio $\pm \mathrm{DE}$ & \\
\hline Edad (años) & $18.2 \pm 0.7$ & $17-20$ & $18.2 \pm 0.5$ & $17-19$ & $18.2 \pm 0.6$ & $17-20$ \\
\hline Peso (kg) & $66.2^{\star \star} \pm 8.7$ & $52-94$ & $57.1 \pm 7.7$ & $47-79$ & $61.3 \pm 9.4$ & $47-94$ \\
\hline Talla (cm) & $173.5^{\star \star} \pm 7.0$ & $160-188$ & $160.5 \pm 5.3$ & $147-174$ & $166.5 \pm 9.0$ & $147-188$ \\
\hline $\mathrm{IMC}(\mathrm{kg} / \mathrm{m} 2)$ & $22.0 \pm 2.6$ & $18-29$ & $22.1 \pm 2.7$ & $17-30$ & $22.1 \pm 2.6$ & $17-30$ \\
\hline$\%$ grasa corporal & $9.9^{\star \star} \pm 4.9$ & $4-23$ & $24.5 \pm 5.4$ & $13-38$ & $17.7 \pm 8.9$ & $4-38$ \\
\hline Cintura (cm) & $74.1^{\star \star} \pm 5.8$ & $64-89$ & $67.1 \pm 8.1$ & $58-112$ & $70.4 \pm 7.9$ & $58-112$ \\
\hline ICC & $0.80^{\star *} \pm 0.0$ & $0.7-0.9$ & $0.70 \pm 0.0$ & $0.6-0.8$ & $0.74 \pm 0.1$ & $0.6-0.9$ \\
\hline Vo2 max (ml/kg) & $40.9^{\star} \pm 7.6$ & $27-65$ & $37.9 \pm 7.7$ & $26-59$ & $39.3 \pm 7.7$ & $26-65$ \\
\hline Glicemia (mg/dl) & $87.2 \pm 11.3$ & $68-114$ & $84.0 \pm 10.6$ & $67-105$ & $85.5 \pm 11.0$ & $67-114$ \\
\hline Colesterol total (mg/dl) & $152.9^{\star \star} \pm 31.2$ & $89-223$ & $181.9 \pm 27.8$ & $124-240$ & $168.6 \pm 32.7$ & $89-240$ \\
\hline LDL (mg/dl) & $95.7^{\star \star} \pm 27.5$ & 40-159 & $120.4 \pm 24.7$ & $65-171$ & $109.3 \pm 28.8$ & $40-171$ \\
\hline HDL (mg/dl) & $37.8 \pm 9.5$ & $28-72$ & $41.0 \pm 8.5$ & $28-70$ & $39.5 \pm 9.0$ & $28-72$ \\
\hline Triglicéridos (mg/dl) & $94.9 \pm 53.7$ & $32-345$ & $99.0 \pm 40.1$ & $39-239$ & $97.2 \pm 46.7$ & $32-345$ \\
\hline Acido Úrico (mg/dl) & $4.9^{\star \star} \pm 1.2$ & $2.7-8.7$ & $3.5 \pm 1.0$ & $1.8-6.9$ & $4.1 \pm 1.3$ & $1.8-8.7$ \\
\hline Sodio plasmático (mEq/L) & $140.3 \pm 2.3$ & $135-146$ & $140.1 \pm 2.6$ & $133-147$ & $140.2 \pm 2.5$ & 133-147 \\
\hline Potasio plasmático (mEq/L) & $4.5^{\star} \pm 0.4$ & $3.8-5.5$ & $4.3 \pm 0.4$ & $3.4-5.2$ & $4.4 \pm 0.4$ & $3.4-5.5$ \\
\hline
\end{tabular}




\section{Cuadro 2 \\ Ingesta diaria de nutrientes de los estudiantes universitarios según sexo. Universidad de Costa Rica, 1996}

\begin{tabular}{|c|c|c|c|c|c|c|}
\hline \multirow[t]{2}{*}{ Ingesta diaria } & \multicolumn{2}{|l|}{ Hombres $(n=51)$} & \multicolumn{2}{|c|}{ Mujeres $(n=59)$} & \multicolumn{2}{|c|}{ Total $(n=110)$} \\
\hline & Promedio $\pm \mathrm{DE}$ & Rango & Promedio $\pm \mathrm{DE}$ & Rango & Promedio $\pm \mathrm{DE}$ & Rango \\
\hline Calorías & $3046.9^{\star \star} \pm 1203.5$ & $927-5913$ & $2069.2 \pm 714.5$ & $996-4732$ & $2522.5 \pm 1084.4$ & $927-5913$ \\
\hline \% proteínas & $14.7 \pm 2.8$ & $9-23$ & $14.9 \pm 4.1$ & $7-26$ & $14.8 \pm 3.5$ & $7-26$ \\
\hline$\%$ carbohidratos & $57.1 \pm 9.6$ & $36-79$ & $54.8 \pm 11.3$ & $29-89$ & $55.9 \pm 10.6$ & $29-89$ \\
\hline$\%$ grasas & $28.9 \pm 8.7$ & $8-49$ & $31.9 \pm 9.2$ & $9-58$ & $30.5 \pm 9.1$ & $8-58$ \\
\hline$\%$ Acido graso saturado & $8.7 \pm 3.7$ & $0.4-17$ & $9.8 \pm 3.9$ & $3-22$ & $9.4 \pm 3.8$ & $0,4-22$ \\
\hline$\%$ Acido graso monoinsaturado & $9.9^{\star} \pm 3.4$ & $0.2-16$ & $11.6 \pm 3.6$ & $3-23$ & $10.8 \pm 3.6$ & $0,2-23$ \\
\hline$\%$ Acido graso poliinsaturado & $4.9 \pm 2.8$ & $0.6-13$ & $5.5 \pm 3.0$ & $1-16$ & $5.2 \pm 2.9$ & $0.6-16$ \\
\hline Colesterol (g/1000 kcal) & $121.1 \pm 80.7$ & $0-369$ & $122.0 \pm 85.3$ & $0-429$ & $121.5 \pm 82.8$ & $0-429$ \\
\hline Fibra (g/1000 kcal) & $5.7 \pm 3.1$ & $1-19$ & $6.5 \pm 3.8$ & $0.7-18$ & $6.1 \pm 3.5$ & $0.7-19$ \\
\hline$B_{12}(\mu \mathrm{g})$ & $5.6^{\star} \pm 4.0$ & $0-16$ & $4.1 \pm 2.3$ & $0.01-9.6$ & $4.8 \pm 3.2$ & $0-16$ \\
\hline$B_{6}(\mu \mathrm{g})$ & $2.3^{*} \pm 1.1$ & $0.8-5.2$ & $1.7 \pm 1.0$ & $0.5-4.2$ & $2.0 \pm 1.1$ & $0.5-5.2$ \\
\hline$\beta$ caroteno $(\mu \mathrm{g})$ & $962.3 \pm 1218.1$ & $3-5125$ & $987.4 \pm 1303.2$ & $3-6170$ & $975.8 \pm 1258.7$ & $3-6170$ \\
\hline Vitamina C (mg) & $209.4 \pm 197.0$ & $4-1141$ & $195.7 \pm 208.6$ & $24-1307$ & $202.0 \pm 202.5$ & $4-1307$ \\
\hline Vitamina $E(m E q)$ & $18.3 \pm 16.6$ & $0-75$ & $15.6 \pm 13.4$ & $0.4-59$ & $16.9 \pm 15.0$ & $0-75$ \\
\hline Acido Fólico $(\mu \mathrm{g})$ & $583.4^{\star \star} \pm 347.7$ & $146-1864$ & $391.4 \pm 225.6$ & $84-1157$ & $480.4 \pm 302.9$ & $84-1864$ \\
\hline
\end{tabular}

plasmáticas bajas de HDL. Por último, todos los siguientes parámetros: el colesterol total, las LDL, la cintura, el IMC, la cantidad ingerida de $\mathrm{B}_{6}$, la fibra, el ácido fólico y la vitamina $\mathrm{C}$ se relacionaron directa y significativamente con los niveles de triglicéridos.

También se calcularon los coeficientes de correlación parcial controlando por el IMC y la ingesta calórica total. Se mantuvieron significativas las siguientes correlaciones: entre el colesterol total y las LDL, los triglicéridos, el porcentaje de grasa y el ICC; entre los niveles de LDL y el colesterol total, los triglicéridos, el porcentaje de grasa, el ICC y el Vomax; entre los niveles de triglicéridos y el colesterol total, las LDL, el consumo de fibra, de ácido fólico y vitamina C. Ninguna de las correlaciones para HDL mantuvo la significancia estadística después del control.

Los modelos de regresión múltiple para las variables del perfil lipídico (colesterol total, LDL, log HDL y log triglicéridos) se muestran en el Cuadro 4. Debido a la elevada correlación que existe entre los niveles de colesterol total y los de LDL, los primeros no se tomaron en cuenta para construir los modelos para las LDL, las HDL ni los triglicéridos, y los segundos no se tomaron en cuenta para construir el modelo para el colesterol total.

Con respecto a los niveles de colesterol total, el modelo incluyó cuatro parámetros que, de manera significativa e independiente, predicen estos valores. Como se puede observar, casi un $50 \%\left(\mathrm{R}^{2}=0.459\right)$ de la variabilidad del colesterol, es explicado por el sexo y los niveles plasmáticos de triglicéridos, HDL y potasio. El modelo para LDL es semejante al del colesterol total pero el $\mathrm{R}^{2}$ es algo menor y además no incluye como variable de predicción los niveles de HDL. Con respecto a los niveles de HDL y triglicéridos, la cintura y la ingesta de $\mathrm{B}_{6}$ predicen los primeros y los niveles de LDL, el IMC y también la ingesta de $\mathrm{B}_{6}$ predicen los segundos.

\section{Discusión}

Los niveles sanguíneos de colesterol, al momento del nacimiento, son muy similares en todas las poblaciones ${ }^{27} \sin$ embargo, posteriormente, la interacción entre una serie de factores ambientales y el genotipo de cada individuo produce una gran variabilidad en el perfil lipídico.

En este trabajo se presentan los factores antropométricos, bioquímicos y dietéticos que durante la juventud se relacionan con los niveles séricos de colesterol total, de LDL, de HDL y de triglicéridos.

El porcentaje de grasa corporal, se asoció, fuertemente y de manera directa con niveles aumentados de colesterol total y LDL. Además, la distribución central de la grasa se relacionó con niveles disminuidos de HDL. Por otro lado, un IMC alto, se asoció con niveles elevados de triglicéridos. De hecho, el IMC formó parte de las variables que, de acuerdo con el modelo de regresión lineal, predicen los niveles plasmáticos de triglicéridos. Estos hallazgos coinciden con los encontrados en el estudio de Bogalusa, ${ }^{27}$ el cual reporta que el peso, la adiposidad y la distribución de grasa no solo se relacionan con niveles séricos alterados de lípidos y lipoproteínas sino que también contribuyen en una presentación temprana de resistencia a la insulina. Así mismo, Daniels y 


\begin{tabular}{|c|c|c|c|c|}
\hline \multicolumn{5}{|c|}{$\begin{array}{c}\text { Cuadro } 3 \\
\text { Coeficientes de correlación de Pearson. } \\
\text { Universidad de Costa Rica, } 1996\end{array}$} \\
\hline Características & Colesterol & LDL & $\overline{\mathrm{HDL}}$ & glicéridos \\
\hline IMC & 0.05 & 0.02 & $-0.20^{*}$ & $0.25^{\star \star}$ \\
\hline$\%$ grasa corporal & $0.40^{* *}$ & $0.39^{\star \star *}$ & 0.06 & 0.14 \\
\hline Cintura & -0.10 & -0.12 & $-0.24^{*}$ & $0.22^{*}$ \\
\hline ICC & $-0.41^{* *}$ & $-0.40^{\star *}$ & $-0.19^{\star}$ & -0.03 \\
\hline VO2 máximo & -0.18 & $-0.22^{\star}$ & 0.05 & 0.00 \\
\hline Glicemia & 0.02 & 0.04 & -0.11 & 0.05 \\
\hline Colesterol & 1.00 & $0.93^{\star \star}$ & 0.17 & $0.47^{\star \star}$ \\
\hline LDL & $0.93^{\star \star}$ & 1.00 & -0.06 & $0.27^{\star \star}$ \\
\hline HDL & 0.17 & -0.06 & 1.00 & $-0.20^{*}$ \\
\hline Triglicéridos & $0.47^{\star \star}$ & $0.27^{\star \star}$ & $-0.20^{*}$ & 1.00 \\
\hline Ácido úrico & -0.14 & -0.13 & $-0.23^{*}$ & 0.12 \\
\hline Sodio plasmático & 0.07 & 0.11 & -0.09 & 0.01 \\
\hline Potasio plasmático & 0.09 & 0.07 & 0.00 & 0.11 \\
\hline Calorías ingeridas & -0.03 & -0.03 & -0.11 & 0.09 \\
\hline$\%$ proteínas & 0.02 & 0.03 & -0.09 & 0.09 \\
\hline$\%$ carbohidratos & -0.13 & -0.11 & -0.09 & -0.04 \\
\hline$\%$ grasas & 0.15 & 0.13 & 0.16 & -0.02 \\
\hline \% ácido graso saturado & 0.09 & 0.11 & 0.16 & -0.15 \\
\hline \% ácido graso monoinsaturado & 0.13 & 0.12 & 0.17 & -0.10 \\
\hline \% ácido graso poliinsaturado & 0.00 & 0.01 & 0.04 & -0.09 \\
\hline Colesterol ingerido & -0.07 & -0.04 & -0.13 & 0.03 \\
\hline Fibra & 0.09 & 0.06 & -0.17 & $0.23^{*}$ \\
\hline B12 & 0.04 & 0.06 & -0.09 & 0.05 \\
\hline B6 & -0.05 & -0.07 & $-0.20^{*}$ & $0.20^{*}$ \\
\hline Beta caroteno & -0.02 & -0.01 & -0.04 & 0.02 \\
\hline Vitamina C & 0.05 & -0.04 & -0.02 & $0.26^{\star \star}$ \\
\hline Vitamina E & 0.00 & -0.04 & 0.03 & 0.04 \\
\hline Ácido fólico & -0.01 & -0.07 & -0.16 & $0.27^{*}$ \\
\hline
\end{tabular}

fican, por medio de relaciones de riesgo y correlaciones intraclase, el agrupamiento significativo de los siguientes parámetros, cuando estos se localizaron sobre el percentilo 75: el IMC, la relación TG/HDL y el índice de resistencia a la insulina.

Con respecto al sexo, se encontró que las mujeres poseen un colesterol total y un nivel de LDL significativamente mayores que los hombres. Tanto en nuestro país, ${ }^{32}$ como fuera, ${ }^{26,33}$ se han reportado, para mujeres entre los 17 y 20 años de edad, que no utilizan gestágenos orales, niveles de colesterol total y de LDL ligeramente $(0$ a $10 \mathrm{mg} / \mathrm{dL})$ superiores a los de los hombres de la misma edad. Nuestros datos revelan una diferencia de $30 \mathrm{mg} / \mathrm{dL}$ para el colesterol total y de $25 \mathrm{mg} / \mathrm{dL}$ para las LDL. Aunque no contamos con una explicación para este hecho, lo preocupante es que coloca a las mujeres en una situación de mayor riesgo, sobre todo porque, en este caso, sus niveles de HDL no son significativamente superiores a los niveles de los hombres. El hallazgo anterior podría encontrar una explicación parcial en la ingesta levemente superior (no estadísticamente significativa) de grasas totales y grasas saturadas de las mujeres. Sin embargo, la relación entre el consumo de ácidos grasos poliinsaturados/saturados fue el mismo en ambos sexos. Debe destacarse que en nuestro estudio, solo dos mujeres reportaron el uso de gestágenos orales.

En relación con las variables dietéticas, se encontró que tanto los hombres como las mujeres comen, diariamente, más de $100 \mathrm{mg}$ de colesterol por cada $1000 \mathrm{kcal}$ ingeridas. Además, en el caso de los hombres, el promedio de la ingesta absoluta diaria de colesterol superó los $300 \mathrm{mg}$. Sin embargo,

cols, en un estudio reciente, realizado en niños y adolescentes, y que incluyó tanto hombres como mujeres, reportan que la distribución central de la grasa o también llamado patrón tipo androide, se asoció con concentraciones plasmáticas de lípidos y lipoproteínas desfavorables. ${ }^{28}$

Dentro del perfil lipídico, deben destacarse los bajos niveles de HDL tanto en hombres como en mujeres. Un estudio de metaanálisis ${ }^{29}$ demostró que las dietas ricas en carbohidratos, en sustitución de grasas saturadas, producen una disminución de las HDL. Este no es el caso de nuestros jóvenes, ya que en ellos sus ingestas de carbohidratos no superaron el $60 \%$ del aporte calórico total diario. Por otro lado, se halló una correlación inversa y significativa entre las HDL y el IMC, el ICC y los niveles plasmáticos de triglicéridos. Esta correlación se podría explicar por la existencia desde edades tempranas de una cierta resistencia a la insulina, lo cual no fue evaluado en esta investigación. Al respecto, Tenkanen y cols., como parte del estudio de Helsinki, encontraron que un $77 \%$ de los individuos con niveles de HDL inferiores a $31 \mathrm{mg} / \mathrm{dl}$ presentaron concentraciones de triglicéridos iguales o superiores a $204 \mathrm{mg} / \mathrm{dL} .{ }^{30}$ Más aún, Chen y cols., ${ }^{31}$ como parte del estudio de Bogalusa, identi- los coeficientes de correlación entre la cantidad ingerida de colesterol y los niveles plasmáticos de colesterol total y LDL no fueron significativos. Al respecto, otras investigaciones tampoco han demostrado una relación directa y significativa entre estas dos variables, de hecho, se ha concluido que la modificación que ejerce la ingesta de colesterol sobre sus propios niveles plasmáticos presenta una considerable variabilidad entre los diferentes individuos. ${ }^{26,27}$

Tanto los niveles de HDL como los de triglicéridos, se relacionaron con la ingesta de $\mathrm{B}_{6}$ (Cuadro 4); los primeros en forma inversa y los segundos de manera directa. No se encontraron otros trabajos que apoyen o contradigan estos hallazgos. Metabólicamente, se podría proponer que los niveles aumentados de $\mathrm{B}_{6}$ estimulen la transaminación de ciertos aminoácidos, lo que conduce a una mayor producción de ácido pirúvico que eventualmente se desviaría a la producción de ácidos grasos y triglicéridos. Esta hipótesis se vería reforzada por un ambiente endocrino y metabólico donde existe cierto grado de resistencia a la insulina, aspecto que merece ser estudiado en el futuro. 


\section{Cuadro 4 \\ Modelos de regresión múltiple escalonada para los componentes del perfil lipídico. Universidad de Costa Rica, 1996}

\begin{tabular}{lllc}
\hline Variable & $\mathrm{R}^{2}$ total & $\begin{array}{l}\text { Variables que } \\
\text { ingresaron en el } \\
\text { modelo }(\mathrm{p}<0.05)\end{array}$ & $\begin{array}{c}\text { Coeficiente } \\
\text { Beta }\end{array}$ \\
\hline Colesterol total & \multirow{2}{0.459}{} & Triglicéridos & 0.460 \\
& & Sexo & -0.419 \\
& & HDL & 0.205 \\
LDL & \multirow{2}{*}{0.282} & Potasio sérico & 0.156 \\
& & Sexo & -0.466 \\
Log HDL & \multirow{2}{*}{0.087} & Potasióridos & 0.216 \\
& & Cintura & 0.183 \\
Log Triglicéridos & $\mathrm{B}_{6}$ & -0.250 \\
& 0.176 & LDL & -0.196 \\
& & IMC & 0.334 \\
& & $\mathrm{~B}_{6}$ & 0.246 \\
& & 0.206 \\
\hline
\end{tabular}

Para construir los modelos se tomaron en cuenta todos los parámetros incluidos en el Cuadro 1, a excepción de las calorías totales ingeridas, los niveles de LDL para el colesterol y los niveles de colesterol para LDL, HDL y triglicéridos.

Otro hallazgo de la dieta, que llama la atención, es la baja ingesta de fibra, encontrada tanto en los hombres como en las mujeres; lo que probablemente refleja el bajo consumo de frutas y vegetales que presentaron, en general, todos los jóvenes estudiados, perdiéndose así el efecto benéfico que ejerce la fibra sobre otros factores asociados con la EAC. ${ }^{34}$

Con respecto a las variables bioquímicas, los modelos de regresión señalaron la concentración sérica de potasio como variable independiente y predictora de los niveles de colesterol total y LDL, por otro lado, se encontró una correlación inversa y significativa entre los niveles plasmáticos de ácido úrico y los niveles de HDL. Estos hallazgos son de gran relevancia y ameritan un enfoque en esta discusión. Se ha considerado la hiperuricemia como un factor predictivo para desarrollar tanto HTA como EAC. ${ }^{35}$ Posiblemente, lo anterior también tiene relación con el síndrome de resistencia a la insulina, en el cual coexisten, entre otros hallazgos, la hiperlipidemia con la hiperuricemia. Una explicación para este fenómeno, se puede encontrar en la observación de alteraciones en la vía glicolítica, como lo describe Leyva y cols. ${ }^{36}$ Ellos postulan una disminución en la actividad de la enzima gliceraldehido3-fosfato deshidrogenasa, la cual es regulada por la insulina. La disminución en la actividad de esta enzima provoca la acumulación de compuestos intermedios y su desviación hacia la producción de ácido úrico y lípidos. La tendencia a presentar mayores niveles plasmáticos de potasio, entre mayores fueran los niveles de colesterol total y LDL, también podría tener relación con el efecto de la insulina sobre el ingreso de este ión en las células blanco de esta hormona.
Por último, se debe enfatizar la relación encontrada entre el $\mathrm{Vo}_{2} \max$ y las variables del perfil lipídico. Se encontró una relación inversa y significativa entre el $\mathrm{Vo}_{2}$ max y los niveles de LDL. Lo anterior pone de manifiesto la importancia del ejercicio físico en la prevención de la EAC, tal y como ha sido reportado previamente..$^{16,37,38}$

El conocimiento de los factores asociados con un perfil lipídico alterado, desde la juventud, podría ayudar a aclarar la naturaleza de la EAC y a una mejor definición de políticas con respecto a la prevención y el manejo de esta enfermedad.

Los resultados de este trabajo ponen de manifiesto la importancia de investigar la presencia de varios factores de riesgo desde edades tempranas y no focalizar la atención en uno de ellos. Los principales hallazgos encontrados apuntan a la coexistencia desde edades tempranas de alteraciones metabólicas que podrían estar genéticamente relacionadas, como ocurre en el síndrome de resistencia a la insulina. El seguimiento de este grupo de jóvenes ayudará a esclarecer puntos importantes sobre la fisiopatología y la epidemiología de la EAC.

\section{Abstract}

Background and objectives: It has been estimated that coronary heart disease, as well as other chronic diseases, has its origins in infancy and adolescence. The purpose of this study was to evaluate, in Costa Rican young students, the relation of anthropometric and biochemical parameters, fitness level and dietary composition to the serum lipid profile (total cholesterol, LDL, HDL and triglycerides)

Population and methods: A sample of 110 youths (59 female and 51 male) between ages 17 and 20 years, were randomly selected from the total of new students that initiated at the University of Costa Rica in 1996. Pearson correlation coefficients were first used to evaluate associations between the lipid profile and anthropometric, biochemical, dietetic and fitness parameters. A stepwise regression procedure was used to identify independent associations between the variables.

Results: Total serum cholesterol and LDL levels correlate positively and significantly $(\mathrm{p}<0.01)$ with percent body fat and triglyceride levels. LDL was inversely associated with maximum oxygen consumption $(\mathrm{p}<0.05)$. Greater levels of triglycerides, uric acid, waist, body mass index and $\mathrm{B}_{6}$ intake were significantly $(\mathrm{p}<0.05)$ associated with lower HDL concentrations. Approximately $50 \%\left(\mathrm{R}^{2}=0.459\right)$ of the cholesterol variability is explained by sex, triglyceride, HDL, and potassium serum levels.

Conclusions: Since adolescence, sex and anthropometric variables like body mass index, waist/hip relation and percent body fat, presented the greater associations with lipid and lipoprotein serum levels. Also, maximum oxygen consumption, uric acid and potassium plasmatic levels, fiber, $\mathrm{B}_{6}$, vitamin $\mathrm{C}$ and folic acid intake were significantly correlated with the lipid profile.

Key words: Coronary disease, risk factors, blood cholesterol, diet, adolescence. 


\section{Referencias}

1. Castelli WP, Garrison RJ, Wilson WPF, Abbott RD, Kalousdian S, Kannel WB. Incidence of coronary heart disease and lipoprotein cholesterol levels; The Framinghan Study. JAMA 1986; 256: 2835-2838.

2. Solberg LA. Strong JP. Risk factors and atherosclerotic lesions: a review of autopsy studies. Arteriosclerosis 1983; 3: 187-198.

3. Lavie CJ. Lipid and lipoprotein fractions and coronary artery disease. Mayo Clin Proc 1993; 68: 618-619.

4. Stamler J, Dyer AR, Shekelle RB, Neaton J, Stamler R. Relationship of baseline major risk factors to coronary and all-cause mortality, and to longevity: findings from long-term follow-up of Chicago cohorts. Cardiology 1993; 82: 191-222.

5. O'Keefe JH, Lavie CJ, McCallister BD. Insights into the pathogenesis and prevention of coronary artery disease. Mayo Clin Proc 1995; 70: 69-79.

6. McGill HC, McMahan CA. Determinants of atherosclerosis in the young. Pathobiological determinants of atherosclerosis in the youth (PDAY) research group. Am J Cardiol 1998; 82: 30T-36T.

7. Berenson GS, Srinivasan SR, Bao W, Newman WP, Tracy RE. Association between multiple cardiovascular risk factors and atherosclerosis in children and young adults. N Engl J Med 1998; 338: 1650-1656.

8. Mahoney LT, et al. Coronary risk factors measured in childhood and young adult life are associated with coronary artery calcification in young adults: The Muscatine Study. J Am Coll Cardiol 1996; 27: 277-84.

9. Webber LS, Srinivasan SR, Wattigney WA, Berenson GS. Tracking of serum lipids and lipoproteins from childhood to adulthood. The Bogaluda Heart Study. Am J Epidemiol 1991; 133: 884-889.

10. Lauer RM, Lee J, Clarke WR. Factors affecting the relationship between chilhood and adult cholesterol levels: the Muscatine Study. Pediatrics 1988; 82: 309-318.

11. Porkka KVK, Viikari J, Akerblom HK. Tracking of serum HDL-cholesterol and other lipids in chilhood and adolescents. The cardiovascular Risk in Young Finns Study. Prev Med 1991; 20: 713-724.

12. Fuster V, Gotto AM, Libby P, Loscalzo J, McGill H. Task Force 1. Pathogenesis of Coronary Disease: The Biologic Role of Risk Factors. J Am Coll Cardiol 1996; 27: 965-976.

13. Hayes KC, Khosla P. Dietary fatty acids and cholesterolemia. FASEB J 1992; 6: 2600-2607.

14. Mensik RP, Katan MB. Effect of dietary fatty acids on serum lipids and lipoproteins. A meta-analysis of 27 trials. Arterioscler Thromb 1992; 12: 911-919.

15. Keys A, Anderson JT, Grande F. Serum cholesterol response to changes in the diet. Metabolism 1965; 14: 776-787.

16. Durstine LJ, Haskell WL. Effects of exercise training on plasma lipids and lipoproteins. Ex Sport Sci Rev 1994; 22: 477-521.

17. Khoury P, Morrison JA, Kelly M, Horvitz R, Glueck CJ. Clustering and interrelationships of coronary heart disease risk factors in schoolchildren, ages 6-19. Am J Epidemiol 1980; 112: 524-538.

18. Smoak CG, Burke GL, Webber LS, Harsha DW, Srinivasan SR, Berenson GS. Relation of obesity to clustering of cardiovascular disease risk factors in children and young adults: the Bogalusa Heart Study. Am J Epidemiol 1987; 125: 364-372.

19. De Fronzo RA, Ferrannini E. Insulin resistance: A multi-faceted syndrome responsible for NIDDM, obesity, hypertension, dyslipidemia and atherosclerotic heart disease. Diabetes Care 1991; 14: 173-194.
20. Bao W, Srinivasan SR, Wattigney WA, Berenson GS. Persistence of multiple cardiovascular risk clustering related to syndrome $\mathrm{X}$ from childhood to young adulthood: The Bogalusa Heart Study. Arch Intern Med 1994; 154: 1842-1847.

21. Fernández-Ramírez A, Ulate-Montero G. Factores de riesgo de enfermedades de arteria coronaria en universitarios de 17 a 19 años de edad. Rev Invest Clin 1998; 50: 457-462.

22. Aragón-Vargas LF, Fernández-Ramírez AS. Fisiología del ejercicio: respuestas, entrenamiento y medición. San José: Universidad de Costa Rica, 1995.

23. Castro M. Evaluación del estado nutricional de adultos. San José: Oficina de Publicaciones de la Universidad de Costa Rica, 1992.

24. Whitney EN, Cataldo CB, y Rolfes S. Understanding normal and clinical nutrition. 3th ed. St. Paul: West Publishing Company, 1994.

25. Montero G. Valoración del uso de fotografías para estimar el tamaño de porción de alimentos. Tesis de Licenciatura en Nutrición. Escuela de Nutrición. Universidad de Costa Rica. 1996.

26. National Cholesterol Education Program. Report of the Expert Panel on Blood Cholesterol Levels in Children and Adolescents. Pediatrics 1992; 89:537-544.

27. Berenson GS, et al. Cardiovascular risk in early life: The Bogalusa Heart Study. Current Concepts. 1991: 41-53.

28. Daniels SR, Morrison JA, Sprecher DL, Khoury P, Kimbal TR. Association of body fat distribution and cardiovascular risk factors in children and adolescents. Circulation 1999; 99: 541-545.

29. Hegsted DM, Ausman LM, Johnson JA, Dallal GE. Dietary fat and serum lipids: an evaluation of the experimental data. Am J Clin Nutr $1993 ; 57: 875-873$

30. Tenkanen L, Pietila K, Manninen V, Mantarri M. The triglyceride issue revisited. Arch Intern Med 1994; 154: 2714-2720.

31. Chen W, Bao W, Begum S, Elkasabany A, Srinivasan SR, Berenson GS. Age-related patterns of the clustering of cardiovascular risk variables of Syndrome $\mathrm{X}$ from childhood to young adulthood in a population made of black and white subjects. The Bogalusa Heart Study. Diabetes 2000; 49: 1042-1048.

32. Monge R, Muñoz L, Faiges F, Rivero A, Alvarado J. Perfil lipídico de adolescentes urbanos costarricenses. Alimentos y Salud 1996; (2): 48.

33. Scriver CR, Beaudet AL, Sly WS, Valle D. The metabolic and molecular bases of inherited disease. $7^{\circ}$ ed. New York: McGraw-Hill, Inc., 1995.

34. Rexrode KM, Manson JE. Antioxidants and coronary heart disease: observational studies. J Cardiovas Risk 1996; 3: 363-367.

35. Johnson RJ, Kivlighn SD, Kim YG, Suga S, Fogo AB. Reappraisal of the pathogenesis and consequences of hyperuricemia in hypertension, cardiovascular disease, and renal disease. Am J Kidney Dis 1999; 33: 225-234.

36 Leyva F, Wingrove CS, Godsland IF, Stevenson JC. The glycolytic pathway to coronary heart disease: a hypothesis. Metabolism 1998; 47: 657-662.

37. Rimmer JH, Looney MA. Effects of an aerobic program on the cholesterol levels of adolescents. RQES 1997; 68: 74-79.

38. Armstrong N, Simons-Morton B. Physical activity and blood lipids in adolescents. Pediatr Exerc Sci 1994; 6: 381-405. 